\title{
Enacted Multiple Representations of Calculus Concepts, Student Understanding and Gender
}

\author{
Ileana Vasu
}

Multiple representations of mathematical ideas can provide students with a better understanding of mathematical concepts (Janvier, 1987). Well-chosen representations are powerful at conveying mathematical concepts. They can be effective at the novice level or for students who perceive themselves as weak in math. They may also provide access to mathematical concepts for those students who lack operational expertise.

Despite interest in multiple representations as a trademark of mathematical success, Calculus instruction is mostly symbolic in nature and lacks consideration of gender specific issues. The gender make-up of the students who opt out of STEM majors after taking Calculus is disproportionately female (Bressoud, 2011; Rassmussen, 2012). Many studies in mathematics education report student difficulties with multiple representations in Calculus but do not explore connections with the enacted curriculum.

Workshop Activities: In this workshop participants shared ideas about mathematical representations in the curriculum as they explored their connections with student understanding and gender. Questions addressed by participants include:

1. How do representations appear in the enacted curriculum and in assessments in Calculus?

2. What is the link between student experience with multiple representations and student understanding?

3. If experience with multiple representations is necessary for a deeper understanding, how should they be incorporated in the curriculum?

4. How do female students relate their learning to multiple representations?

I. Vasu $(\bowtie)$

Holyoke Community College and University of Massachusetts, Amherst, MA, USA

e-mail: ivasu@educ.umass.edu; ivasu@hcc.edu

(C) The Author(s) 2017

G. Kaiser (ed.), Proceedings of the 13th International Congress on Mathematical

Education, ICME-13 Monographs, DOI 10.1007/978-3-319-62597-3_138 
To tighten the discussion, participants were asked to examine Calculus exams from various colleges for the presence and quality of representations. Then, snapshots of Calculus curricula, including the use of multiple representations, the classroom culture and discourse, and the pedagogical approaches were then presented to the participants, in conjunction with videos of students in these curricula as they were solving Calculus problems.

Trends: Participants then fleshed out common themes, patterns, and emergent ideas based on the videotapes and student artifacts. The trends noted were:

- The majority of the assessments were symbolic and procedural. Most exams contained one other representation. No exam contained all representations.

- Student use of multiple representations is aligned with their experience in the classroom, but this is especially true for female students

- Students in the active curricula we examined, made use of more representations in solving problems, were more able to take risks when thinking about strategies and to back track when they reached areas of conflict.

- Students in the lecture class were limited in the number of strategies they attempted and ignored areas of conflict

- Curricula that use contextual representations help students become more self sufficient and generate multiple methods in solving Calculus problems.

These trends were in agreement with quantitative results obtained by the organizer. The workshop concluded that mathematical rich tasks in active curricula may serve as a catalyst for student understanding of Calculus and discussed possible teaching and research directions.

\section{Reference}

Bressoud, D. (2011). The Calculus I instructor. From Launchings. Retrieved from https://www. maa.org/external_archive/columns/launchings/launchings_06_11.html

Janvier, C. (1987). Translation processes in mathematics education. In C. Janvier Problems of Representations in the Learning and Teaching of Mathematics [Papers derived from a symposium organized by CIRADE of Université du Québec à Montréal] (pp. 27-32). Hillsdale, NJ: L. Erlbaum Associates.

Rasmussen, C. (2012). A report on a national study of college calculus: Who is switching out of STEM and why. Plenary address at the 15th Conference on Research in Undergraduate Mathematics Education, Portland, OR.

Open Access Except where otherwise noted, this chapter is licensed under a Creative Commons Attribution 4.0 International License. To view a copy of this license, visit http://creativecommons. org/licenses/by/4.0/.

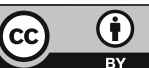

